\title{
Wrist and Grasp Myocontrol: Online Validation in a Goal-Reaching Task
}

\author{
Markus Nowak ${ }^{1 *}$, Beatrice Aretz ${ }^{1}$ and Claudio Castellini ${ }^{1}$
}

\begin{abstract}
Simultaneous and proportional control of hand and wrist prostheses based upon surface electromyography (myocontrol) is still largely an open issue in the community of assistive robotics. It entails the ability of discriminating the activation levels for each degree of freedom (DOF) of the hand/wrist complex, using as few sensors as possible. Furthermore, one should avoid having the human subject train the underlying machine-learning (ML) system with all combinations of activations. In order to tame this problem we have proposed Linearly Enhanced Training (LET), a procedure through which a training set, composed of single-DOF activations provided by the user, is artificially completed with synthetic multi-DOF activations.

In this paper, we validate the $L E T$ procedure through an online psychophysical experiment carried out on 16 intact subjects and one trans-radial amputee, in which a specific goal must be reached within a determined amount of time. Each subject tried to reach the goal in either of four different scenarios, while the $L E T$ procedure was activated or not, and while an optimisation was used or not. A comparative analysis of the results reveals that the usage of $L E T$ does not entail any statistically significant difference in the overall performance, and that the usage of the optimisations significantly improves it. Therefore, one can benefit from the drastic reduction of training time due to $L E T$ without suffering from significant reduction in performance. Furthermore, the optimisation showed the strong tendency to reduce the time it took to successfully accomplish a task, on average by $1.195 \mathrm{~s}$. Furthermore, a comparison of the intact subjects and the trans-radial amputee showed that half of the performance measures of the amputee lie in the $95 \%$ confidence interval determined by the able-bodied group.
\end{abstract}

\section{INTRODUCTION}

After two decades of research, the problem of letting trans-radial (i.e., below-elbow) amputees naturally control a hand/wrist self-powered prosthesis is still open. Specifically, two-degrees-of-freedom (DOF) prosthetic wrists and multifingered prosthetic hands have now appeared on the clinical market, and for such dexterous mechatronic artefacts it is highly desirable [1] that each single DOF of the prosthetic complex be controlled to the desired level of activation. For example, that the wrist be supinated at $50 \%$ of its maximum allowed activation while the hand is grasping a mug with full activation. This kind of control is called simultaneous and proportional [2] and it represents the most advanced vision of how amputees should be able to use their prosthesis.

The technical challenges hidden in the problem are formidable. Traditionally, the information is extracted from the subject via surface electromyography (sEMG [3], [4]) sensors, detecting the degree of intended voluntary activation

\footnotetext{
${ }^{1}$ DLR - German Aerospace Center, D-82234 Weßling, Germany

*Corresponding Author: markus. nowak@dlr.de
}

of a set of muscle remnants in the stump. Such information is evaluated by a machine-learning (ML) approach using a set of samples stored in the beginning of the calibration (training set), producing appropriate control commands for the prosthesis. Even abstracting from the well-known problems of sEMG such as sweat, sensor displacement and muscle fatigue, it is unclear how to unambiguously detect and distinguish the activation of single DOFs from such signals, all at the same time and in real time. Moreover, simultaneous combinations of activations (multi-DOF), such as the one exemplified above, represent a hindrance for the subject. The number of multi-DOF activations is potentially exponential in the number of DOFs of the prosthesis. Nevertheless they must all be accurately produced by the subject so that the training set contains a fair representation of the input space. This is an unacceptable burden placed on the subject. Additionally, specific combinations can be hard to figure out and enforce for amputees, who usually have little or no feedback about what they are trying to do.

In order to alleviate this problem, a few studies have appeared in which a reduced training set is gathered, only containing single-DOF activations (e.g., wrist flexion / extension, wrist pronation / supination and hand opening / closing); subsequently, multi-DOF activations are interpreted as compositions of single-DOF ones. In [5] a fully linear decomposition model was hypothesised. In [6] a mixed model was proposed, in which Euclidean distance in the input space was used to determine whether to classify the current pattern as a single-DOF activation, or to project it onto a linear mixture of them.

In [7]-[9] we proposed to tackle the problem from another point of view, namely, by augmenting the single-DOF training set with an educated guess of artificially produced samples representing multi-DOF activations. The approach, called LET for Linearly Enhanced Training, has shown promising results both when applied on- and offline to multifinger combined activations [7], [8] and when applied offline in hand and wrist movements [9]. In this paper we further validate the technique in a similar online goal-reaching experiment, involving hand and wrist movement. This study was performed involving both intact subjects and a transradial amputee.

\section{Materials And Methods}

The objective of the experiment was to test whether, in an online goal-reaching task, the usage of the LET procedure would produce similar accuracy results when compared to traditional training on a full data set. Furthermore, we 
devised an optimisation to our ML algorithm, and we wanted to check whether this optimisation significantly improved the performance or not. Thus, the experiment consisted of four independent sub-experiments ("scenarios"), in each of which either of these two characteristics (the usage of LET or not, and the usage of the optimisation or not) was enforced in turn. Performance measures were then comparatively assessed.

\section{A. Myocontrol algorithm}

The LET procedure [7]-[9] was devised in order to spare the subject the necessity of producing multi-DOF activations during the training phase. The central idea behind it is the approximation of sEMG data of multi-DOF activations $\left(\boldsymbol{X}_{i j}\right)$ as a combination of the sEMG data of single-DOF activations $\left(\boldsymbol{X}_{i}\right.$ and $\left.\boldsymbol{X}_{j}\right)$.

$$
\boldsymbol{X}_{i j} \approx \mathcal{F}\left(\boldsymbol{X}_{i}, \boldsymbol{X}_{j}\right)
$$

In our case we use a simple linear combination with a single parameter for each multi-DOF activation $\left(\alpha_{i j}\right)$.

$$
\mathcal{F}\left(\boldsymbol{X}_{i}, \boldsymbol{X}_{j}\right)=\alpha_{i j} \cdot\left(\boldsymbol{X}_{i}+\boldsymbol{X}_{j}\right)
$$

These parameters can be determined once for a representative population and thereafter applied to all subsequent users. This has been shown for single- and multi-DOF activations of fingers [8]. In case of combined wrist and grasp activations, the parameter have been determined in a separate experiment [9]. The resulting values are

$$
\begin{array}{lll}
\alpha_{\mathrm{DD} 1}=0.7728 & \alpha_{\mathrm{DD} 2}=0.7741 & \alpha_{\mathrm{DD} 3}=0.4404 \\
\alpha_{\mathrm{DD} 4}=0.8465 & \alpha_{\mathrm{DD} 5}=0.7366 & \alpha_{\mathrm{TD} 1}=0.5377 \\
\alpha_{\mathrm{TD} 2}=0.3980 & &
\end{array}
$$

The acronym in the indices of the $\alpha$-parameters can be found in Table I.

Notice that LET acts on the training set, enriching it with synthetically determined samples representing the multiDOF activations, therefore simplifying the data gathering phase from the subject. The central point here is that, using the $L E T$-enhanced training set, any machine learning method of choice could potentially be used. In particular, we chose Ridge Regression with Random Fourier Features (RRRFF) [10]-[12]. RR-RFF can be seen as a finite-dimensional approximation of a Least-Squares Support Vector Machine using Fourier coefficients. This method provides several advantages. Besides providing the required properties, such as being proportional, the approach is also bound in space. Having a finite number of dimensions makes the algorithm independent of the number of samples used for training. This feature is particularly beneficial in combination with LET. For more details, please refer to the above-mentioned papers.

\section{B. Optimisation}

Typically, in myocontrol it is difficult to measure ground truth when working with amputees, e.g. force for particular grasps. We therefore used the so-called realistic approach
[12], in which the values of the visual stimulus presented to the participant were used as the target values for each sEMG sample. In particular, 1.0 for full activation and 0.0 for no activation were used. Now, due to nature of RRRFF, particularly to the periodicity of the kernel basis functions (cosines), ambiguous prediction results are obtained for activations performed with a higher level of force than the force level used during data collection for training the ML algorithm. (On the other hand, intermediate levels of force are interpreted well.) In fact, activation values of more than 1.0 can easily be reached by the subject, leading to decreasing predicted activation when increasing the actual activation. The left panel of Figure 1 shows a typical case.

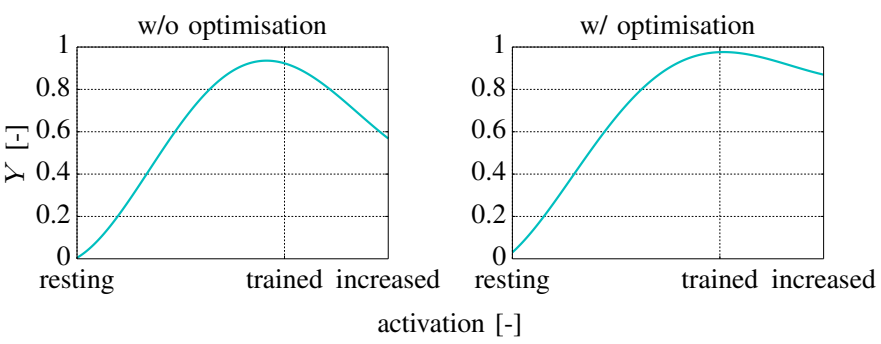

Fig. 1. Prediction $Y$ over increasing level of activation. Level increased stand for $50 \%$ higher activation than during training (trained). (Left panel) without optimisation; (right panel) with optimisation

To counter this behaviour, the data set for training was further enhanced by creating artificial data for higher levels of activation. This optimisation reuses the data gathered during training, which is associated with a full activation, and artificially adds another data cluster for an activation with a $30 \%$ higher level than the full activation. An example of this optimisation for a simple offline case can be found in Figure 1 on the right. The decrease in predicted activation has to be reduced without negatively influencing the continuous regime between rest and trained activation. Furthermore, a threshold on the activation was used for one particular DOF, wrist pronation, to reduce interactions with other DOFs. Predicted activations to the threshold of 0.3 was set to 0.0 . The segment from 0.3 to 1.0 was linearly mapped to $[0.0,1.0]$.

Notice that these optimisations add new samples to the training set (with the underlying assumption that the same machine learning method can be still used) and put a limitation on one of the predicted values after the model has been evaluated. Since the method of choice (see the previous Subsection) is independent of the number of training samples, the cost function associated to the optimised training set remains the same, allowing for a transparent reusal of the technique.

\section{Experimental Setup and Protocol}

The experimental setup is shown in Figure 2. A standard laptop was used for all calculations and execution of the experiment. To acquire sEMG we used the Myo bracelet from Thalmic Labs Inc., which was placed on the proximal end of the forearm, where the muscles bellies can be found. 
TABLE I
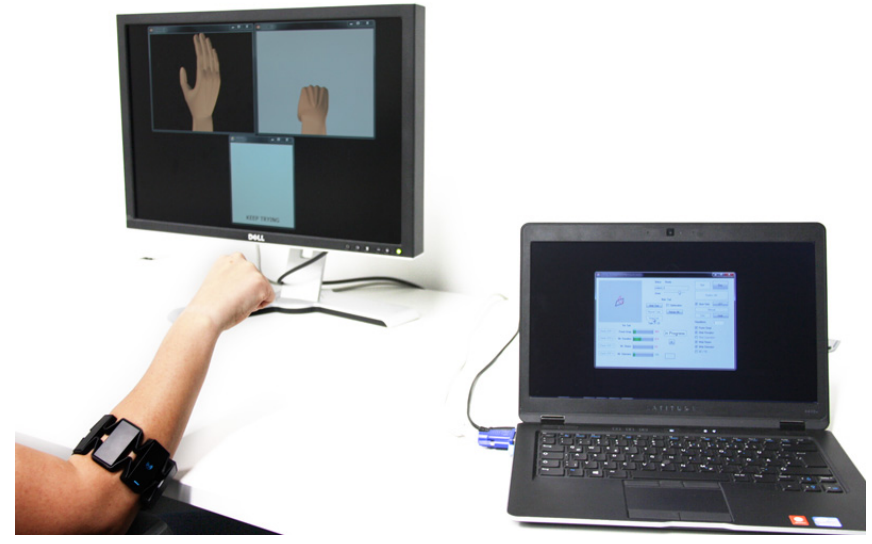

Fig. 2. The experimental setup: a screen depicting the stimulus hand model and the prediction hand model (top left corner), a laptop running the custom software (bottom right corner) and the arm of a subject wearing the Myo bracelet from Thalmic Labs Inc. (bottom left corner).

The connection to the Myo bracelet was established via Bluetooth. The lack of any cable to the laptop gives the participant the maximum freedom in performing the experiment. Among other data, the Myo bracelet provides eight channels of raw sEMG data. The sensors are uniformly distributed along the bracelet. Data from these sensors was sampled at approx. $100 \mathrm{~Hz}$, rectified and further processed by calculating the root-mean-square (RMS) across a moving window of $200 \mathrm{~ms}$. The data was low-pass filtered at $0.2 \mathrm{~Hz}$ using a Butterworth filter of $1^{\text {st }}$ order. This low-pass filtered envelope of the sEMG signal was used as input for the ML algorithm.

Visual cues for the subject were provided on an external screen in form of virtual hand models, one on a grey background depicting the desired activation and one on a black background depicting the current prediction of the ML algorithm.

This experiment involved the same four single DOFs of hand and wrist as in the offline experiment in [9], where the $\alpha$-parameters were determined. These are power grasp, wrist pronation, wrist flexion and wrist extension. Due to the mutual exclusion of the latter two there are seven possible combinations of these four single-DOF activations. These can be found in the following Table I. The abbreviation SD, DD and TD stand for single-, double- and triple-DOF.

Two aspects were investigated here: first, the influence of the optimisation on the quality of online wrist and grasp myocontrol; second, the influence of the LET procedure on the quality of said control. Each of the two factors can either be true or false, which results in four possible scenarios. Each participant in the study performed all four of those scenarios. Due to the relative length, the experiment was split in two sessions. LET was varied between sessions, while the optimisation was varied within a session. The reason for this arrangement is the difference in the training phase of the $L E T$ and non-LET scenario. In the traditional full training (nonLET) case all 11 combinations have to be performed during training. The advantage of the $L E T$ procedure consists of the
ALL POSSIBLE SINGLE- AND MULTI-DOF COMBINATIONS PERFORMED IN THIS EXPERIMENT ALONG WITH THEIR CORRESPONDING ABBREVIATIONS

\begin{tabular}{|c|c|c|c|c|c|}
\hline \# & Pow. Gr. & Wr. Pro. & Wr. Ext. & Wr. Flex. & abbreviation \\
\hline $\begin{array}{l}1 \\
2 \\
3 \\
4\end{array}$ & $\mathrm{X}$ & $\mathrm{X}$ & $\mathrm{x}$ & $\mathrm{X}$ & $\begin{array}{l}\text { SD1 } \\
\text { SD2 } \\
\text { SD3 } \\
\text { SD4 }\end{array}$ \\
\hline $\begin{array}{l}5 \\
6 \\
7 \\
8 \\
9\end{array}$ & $\begin{array}{l}\mathrm{X} \\
\mathrm{X} \\
\mathrm{X}\end{array}$ & $\begin{array}{l}X \\
X\end{array}$ & $\mathrm{x}$ & $\begin{array}{l}\mathrm{X} \\
\mathrm{x}\end{array}$ & $\begin{array}{l}\text { DD1 } \\
\text { DD2 } \\
\text { DD3 } \\
\text { DD4 } \\
\text { DD5 }\end{array}$ \\
\hline $\begin{array}{l}10 \\
11\end{array}$ & $\begin{array}{l}\mathrm{X} \\
\mathrm{X}\end{array}$ & $\begin{array}{l}\mathrm{X} \\
\mathrm{X}\end{array}$ & $\mathrm{x}$ & $\mathrm{X}$ & $\begin{array}{l}\text { TD1 } \\
\text { TD2 }\end{array}$ \\
\hline
\end{tabular}

reduction in training time due to the approximation of the multi-DOF training data. Therefore, in the LET case, only four instead of 11 activations have to be performed, resulting in a much shorter training phase. Training consisted of three repetitions of the respective activations.

In the online goal-reaching experiment two repetitions of these 11 different activations were performed for each of the four scenarios. The goals were one of the 11 different activations at random levels between $30 \%$ and $100 \%$ of full activation for each of the individuals DOFs involved in the activation. The size of the target was $\pm 25 \%$ of the target value for each individual DOF. This results in an approx. target size of $3.7 \%$ of the working space. In order to exit a task successfully the participant had to remain for $1.5 \mathrm{~s}$ consecutively in the target area. For each task the participant had $30 s$ to complete it. After said amount of time elapsed the task was counted as failed. Between one task and the subsequent one, the participants were allowed to rest as long as they intended. Based on this process we are able to determine four characteristic performance measures for each participant and each of the four scenarios. Namely these are, the success rate (SR), the task completion time (TCT), the longest stable time (LST) and the unreachable attempt rate (UA). The SR represents the percentage of successful task. Furthermore, the TCT is the average time that the subject took to successfully exit a task. The UA represents the percentage of unreachable task. Unreachable task are those tasks, where the user was not able to reach the target area at all. All tasks besides successful and unreachable ones are called overshoots. Here the subject was able to reach the target area, but was not able to stably remain in the area for long enough. These tasks are evaluated using the LST. This is the averaged longest duration a subject was able to maintain in the target area. All these measures have been used previously to evaluate myocontrol, similarly or in this exact manner [8], [13].

Sixteen able-bodied subjects ( 3 women and 13 men, age 21 to 30) participated in this experiment. Furthermore, we were able to involve an trans-radial amputee for our study, a 68-year-old man who lost his hand and part of his forearm due to a traumatic event. The amputation was performed 
in 2009 and since then he did not use a prosthesis, neither cosmetic nor myocontrolled. Due to limited time, we were only able to perform a reduced version of the experiment. We only tested the factor $L E T$, while not including the optimisation.

The experiment was approved by the ethics committee of the DLR and followed the Declaration of Helsinki. Each participant was informed beforehand about the process of the experiment and signed an informed consent form.

\section{EXPERIMENTAL RESULTS}

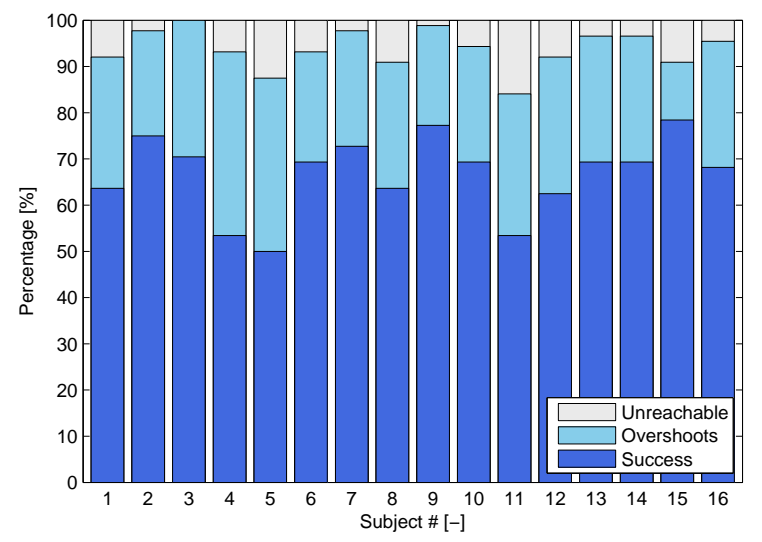

Fig. 3. Relative performance for each of the 16 subjects separated in percentage of successful attempts, overshoots and unreachable attempts. This is a combined view of all four scenarios.

An overview of the experimental results can be found in Figure 3. For each subject, successful attempts, overshoots and unreachable attempts are shown; the bars are mean values across all four scenarios. As one can see, the percentage of successful attempts ranges from $50 \%$ to $78.4 \%$, whereas the percentage of unreachable attempts ranges from $0 \%$ to $15.9 \%$.

A more detailed picture of the results, this time showing the performance measures for each of the four investigated scenarios, can be found in Figure 4. Consider the cyan boxplots versus the blue boxplots: a qualitative inspection of the Figure reveals that the usage of LET slightly decreases the SR (left upper panel), seems to be irrelevant for the TCT and LST (right upper and left lower panel), and slightly increases the UA when the optimisation is turned off. On the other hand - consider now the left-hand boxplots versus the right hand ones in each panel, turning the optimisation on seems to decrease the TCT.

These results were statistically evaluated. We used a twoway repeated measures MANOVA, which corrects for the within-group variance. The two factors were the application of LET and the application of the optimisation. The threshold for significance was set to $\alpha=0.05^{1}$. The results of the two-way repeated measures MANOVA showed that the optimisation improves the performance significantly, while the application of the $L E T$ procedure does not result in

\footnotetext{
${ }^{1}$ Not to be confused with the parameter $\alpha$ involved in the $L E T$ procedure.
}

a significant decrease in performance. The results of the MANOVA are summarised in the following Table II.

TABLE II

RESULTS OF THE TWO-WAY REPEATED MEASURES MANOVA

PERFORMED ON THE FOUR PERFORMANCE MEASURE OF THE 16 INTACT SUBJECTS. WE REPORTED THE MAIN EFFECTS (LET AND

OPTIMISATION) AS WELL AS THE INTERACTION OF THE MAIN EFFECTS.

\begin{tabular}{l||l|l|l|l|l} 
Factor & Pillai's trace & $F$ & $d f$ & error $d f$ & $\mathrm{p}$ \\
\hline \hline LET & 0.40512 & 2.0431 & 4 & 12 & 0.152 \\
\hline optimisation & 0.56648 & 3.9202 & 4 & 12 & $0.0292 *$ \\
\hline interaction & 0.32583 & 1.4499 & 4 & 12 & 0.2774
\end{tabular}

After the combined analysis we performed a uni-variate two-way repeated measures ANOVA for each of the four performance measures individually. Bonferroni correction was applied to the threshold of significance to correct for multiple comparisons, $\alpha_{\text {corr. }}=\frac{\alpha}{m}$, with the number of comparisons $m=4$. The uni-variate analysis of SR shows the opposite behaviour than the multi-variate analysis. The factor LET shows significant interaction $(F(1,15)=9.639, p=$ $0.00725)$, while the factor optimisation does not $(F(1,15)=$ $4.224, p=0.0577)$. TCT follows rather the multi-variate analysis with non-significant interaction for $\operatorname{LET}(F(1,15)=$ $0.156, p=0.698)$. The interaction for optimisation is very close to the threshold $\left(\alpha_{\text {corr. }}=0.0125\right)$, but still nonsignificant $(F(1,15)=7.949, p=0.0129)$. Both, LST and UA, show no significant interaction for neither of both factors in the individual analyses (with $F(1,15)=0.039, p=0.846$ and $F(1,15)=0.091, p=0.767$ for the factors LET and optimisation for LST and $F(1,15)=3.817, p=0.0697$ and $F(1,15)=0.155, p=0.699$ for the factors LET and optimisation for UA).

Furthermore, we determined the $95 \%$ confidence interval for each of the performance measure based on the ablebodied participants. The results can be found in Table III.

TABLE III

95\% CONFIDENCE INTERVAL (CFI) FOR THE 16 ABLE-BODIED PARTICIPANTS COMPARED TO THE RESULTS OF THE SINGLE AMPUTEE PARTICIPANT. HIGHLIGHTED IN GRAY, WHERE THE AMPUTEE PERFORMANCE LIES IN WITHIN THE INTERVAL.

\begin{tabular}{l||c|c|c} 
& measures & $95 \%$ CFI & amputee \\
\hline \hline \multirow{2}{*}{$L E T$} & SR & {$[51.38 \%, 66.81 \%]$} & $27.27 \%$ \\
no optimisation & TCT & {$[10.21 \mathrm{~s}, 12.28 \mathrm{~s}]$} & $17.33 \mathrm{~s}$ \\
& LST & {$[0.6467 \mathrm{~s}, 0.8020 \mathrm{~s}]$} & $0.7762 \mathrm{~s}$ \\
& UA & {$[4.858 \%, 11.05 \%]$} & $9.090 \%$ \\
\hline \multirow{3}{*}{ no $L E T$} & SR & {$[63.65 \%, 76.13 \%]$} & $36.36 \%$ \\
no optimisation & TCT & {$[10.76 \mathrm{~s}, 13.19 \mathrm{~s}]$} & $11.25 \mathrm{~s}$ \\
& LST & {$[0.5857 \mathrm{~s}, 0.8169 \mathrm{~s}]$} & $0.7523 \mathrm{~s}$ \\
& UA & {$[2.198 \%, 7.461 \%]$} & $13.64 \%$
\end{tabular}

This was done to compare the performance of the single amputee participant to the remaining group. When applying the LET procedure the performance of the amputee was within the $95 \%$ CFI for LST and UA. Without the usage of LET, TCT and LST were within the 95\% CFI. These cases are highlighted in gray in Table III. 

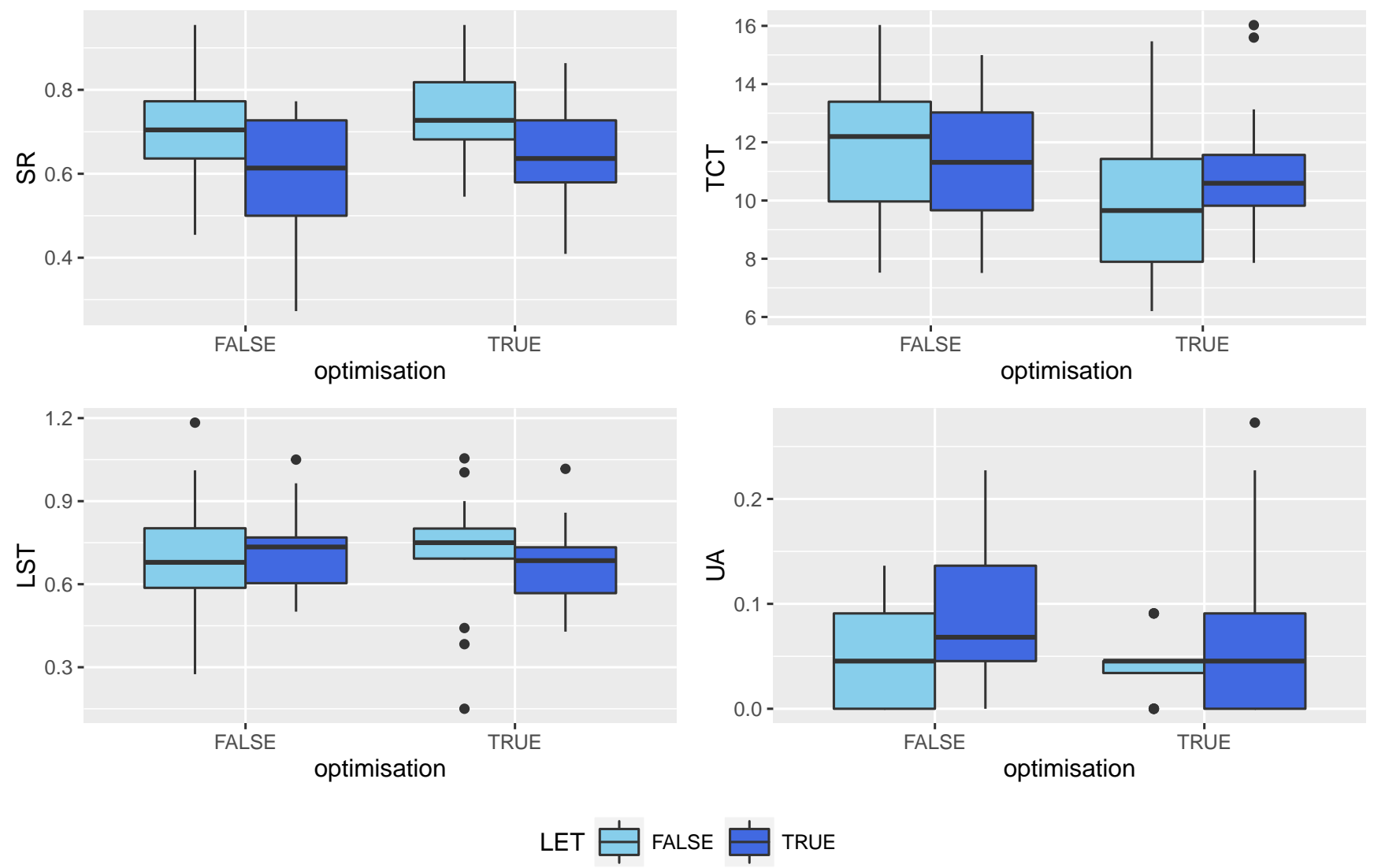

Fig. 4. Boxplot for each of the four performance measures separated according to all four scenarios. Lower and upper hinge of the box represent the $25 \%$ and $75 \%$ percentile, respectively with the band inside the box representing the median. Whiskers extend to the maximum and minimum values within 1.5 $I Q R$, where $I Q R$ is the inter-quartile range. Values outside this range are outliers and are displayed as dots.

\section{DiscuSSION AND CONCLUSIONS}

The multi-variate statistical analysis showed that the application of the optimisation significantly improves the performance, while the LET procedure does not significantly decrease it. This shows us that the cumbersome full training procedure can be reduced to only training single-DOFs with only a limited influence on the performance and therefore reducing the burden on the user. Furthermore, the uni-variate analyses revealed that the application of LET decreased the number of successfully accomplished task (SR) on average by $11.08 \%$. However, the time it took to successfully finish the tasks (TCT) was not influenced by the application of LET. The application of the optimisation on the other hand revealed a tendency to lower the TCT on average by $1.195 \mathrm{~s}$. Both, LST and UA, are not conclusively influenced by neither the application of LET nor of the optimisation. Given these results, we claim that $L E T$ can effectively substitute the full data gathering, and that the optimisation presented can improve the performance further.

Furthermore, a comparison of the 16 able-bodied subjects and the single trans-radial amputee showed that for half of the performance measure both groups performed similarly. The biggest difference can be found in the SR. However, for certain scenarios the task completion time and number of unreachable attempts of the amputee were in the $95 \%$ confidence interval of the able-bodied group. Note, that in the seven years since his amputation the amputee did not use any prosthesis and therefore did not use his forearm muscles. Also, this was the first time he used a myocontrol algorithm. Taking this into account makes the results very promising.

When dealing with a dexterous, multi-DOF hand and wrist prosthesis, it is infeasible to expect an amputated person to produce all possible combinations of the DOF activations during the training phase. To solve this problem we have devised the $L E T$ procedure, which allows in principle to deduce multi-DOF activations starting from single-DOF activations only, thereby reducing the burden on the subjects. In this paper we have presented the results of a psychophysical online experiment, aimed at verifying whether LET could be used as a substitute of the full data gathering, whether it would significantly decrease the performance, and whether an optimisation would improve the situation even more.

Concluding the results, LET is effective in reaching proportional activation goals, which is exactly the objective of simultaneous and proportional control and was tested by randomly chosen targets in the working space. If proved valid in the large, these results pave the way to a uniform approach to simultaneous and proportional control training, 
in which the user only needs to train the ML algorithm on single-DOF activations. The $L E T$-enhanced system will take care of predicting of the remaining multi-DOF activations. Let us remark once again that $L E T$ augments the training set irrespective of what ML algorithm is actually used to build the model, meaning that any method of choice can be employed. On the other hand, the incrementality of the method we chose in this particular experiment (i.e. RRRFF) enables for continuous correction and enhancement of the single-DOF data, involving the subject in a virtuous loop of reciprocal learning. Exploring and exploiting this phenomenon is the next aim of future research.

Further lines of research include, e.g., the application to a real hand/wrist prosthesis such as, e.g., Ottobock's Michelangelo, further testing on a wide population of amputees and exploring further improvements to the proposed optimisation. Given that RR-RFF is strictly bounded in space, there is no limit in principle to the size of the training set to be used. This entails that more strategies to "saturate" the training set could be devised in order to improve the stability of the control.

\section{ACKNOWLEDGMENTS}

We would like to acknowledge Dr. Andreas Arkudas of the Department of Plastic and Hand Surgery at the FriedrichAlexander-University of Erlangen-Nürnberg for putting us in contact with our participant Mr. D.

\section{REFERENCES}

[1] A. Fougner, E. Scheme, A. D. C. Chan, K. Englehart, and Ø. Stavdahl, "A multi-modal approach for hand motion classification using surface emg and accelerometers," in Annual International Conference of the IEEE Engineering in Medicine and Biology Society (EMBC), 2011, pp. 4247-4250.

[2] N. Jiang, J. Vest-Nielsen, S. Muceli, and D. Farina, "EMG-based simultaneous and proportional estimation of wrist/hand kinematics in uni-lateral trans-radial amputees," Journal of NeuroEngineering and Rehabilitation, vol. 9, no. 1, p. 42, 2012.

[3] R. Merletti, A. Botter, C. Cescon, M. Minetto, and T. Vieira, "Advances in surface EMG: Recent progress in clinical research applications," Critical reviews in biomedical engineering, vol. 38, no. 4, pp. 347-379, 2011.

[4] R. Merletti, M. Aventaggiato, A. Botter, A. Holobar, H. Marateb, and T. Vieira, "Advances in surface EMG: Recent progress in detection and processing techniques," Critical reviews in biomedical engineering, vol. 38, no. 4, pp. 305-345, 2011.

[5] N. Jiang, P. Parker, and K. Englehart, "Extracting simultaneous and proportional neural control information for multiple degree of freedom prostheses from the surface electromyographic signal," IEEE Transactions on Biomedical Engineering, vol. 56, no. 4, pp. 1070-1080, 2009.

[6] S. Amsuess, P. Goebel, B. Graimann, and D. Farina, "A multi-class proportional myocontrol algorithm for upper limb prosthesis control: Validation in real-life scenarios on amputees," Neural Systems and Rehabilitation Engineering, IEEE Transactions on, vol. 23, no. 5, pp. 827-836, Sept 2015.

[7] C. Castellini and M. Nowak, "EMG-based prediction of multi-DOF activations using single-DOF training: a preliminary result," in Proceedings of MEC - Myoelectric Control Symposium, 2014, pp. 45-49.

[8] M. Nowak, "The LET procedure for prosthetic myocontrol: towards multi-DOF control using single-DOF activations," Master's thesis, DLR - German Aerospace Center, 2014, available at: http://elib.dlr. de/92975; under review at PLoS ONE with the same title.

[9] M. Nowak and C. Castellini, "Wrist and grasp myocontrol: Simplifying the training phase," in Rehabilitation Robotics (ICORR), 2015 IEEE International Conference on. IEEE, 2015, pp. 339-344.
[10] A. Rahimi and B. Recht, "Random features for large-scale kernel machines," in Advances in Neural Information Processing Systems 20, 2008, pp. 1177-1184.

[11] - "Uniform approximation of functions with random bases," in Allerton Conference on Communication Control and Computing (Allerton08), September 2008, pp. 555-561.

[12] A. Gijsberts, R. Bohra, D. S. González, A. Werner, M. Nowak, B. Caputo, M. A. Roa, and C. Castellini, "Stable myoelectric control of a hand prosthesis using non-linear incremental learning," Frontiers in Neurorobotics, vol. 8, no. 8, pp. 1-15, 2014. [Online]. Available: http://www.frontiersin.org/neurorobotics/10.3389/ fnbot.2014.00008/abstract

[13] N. Jiang, I. Vujaklija, H. Rehbaum, B. Graimann, and D. Farina, "Is accurate mapping of EMG signals on kinematics needed for precise online myoelectric control?" IEEE Transactions on Neural Systems and Rehabilitation Engineering, vol. PP, no. 99, pp. 1-1, 2013. 